ANL-HEP-PR-02-073, EFI-02-56, hep-ph/0209272

\title{
Two-Body Cabibbo-Suppressed Charmed Meson Decays
}

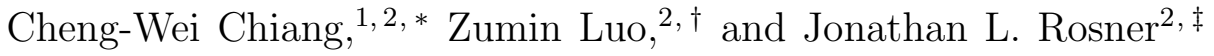 \\ ${ }^{1}$ HEP Division, Argonne National Laboratory 9700 S. Cass Avenue, Argonne, IL 60439 \\ ${ }^{2}$ Enrico Fermi Institute and Department of Physics, \\ University of Chicago, 5640 S. Ellis Avenue, Chicago, IL 60637
}

(Dated: October 29, 2018)

\begin{abstract}
The singly-Cabibbo-suppressed decays of charmed particles governed by the quark subprocesses $c \rightarrow s u \bar{s}$ and $c \rightarrow d u \bar{d}$ are analyzed using a flavor-topology approach, based on a previous analysis of the Cabibbo-favored decays governed by $c \rightarrow s u \bar{d}$. Decays to $P P$ and $P V$, where $P$ is a pseudoscalar meson and $V$ is a vector meson, are considered. We include processes in which $\eta$ and $\eta^{\prime}$ are produced.
\end{abstract}

*e-mail: chengwei@hep.uchicago.edu

$\dagger$ †-mail: zuminluo@midway.uchicago.edu

‡e-mail: rosner@hep.uchicago.edu 


\section{INTRODUCTION}

The decays of charmed particles can provide useful information about strong interactions. The magnitudes and phases of weak couplings governing these decays are well-specified in the standard electroweak theory, so that decay amplitudes serve mainly to illuminate the relative importance of various flavor topologies and their relative strong phases. This has been shown both for Cabibbo-favored decays [1] and more recently for doubly-Cabibbosuppressed processes [2]. In the present article we extend these analyses to singly-Cabibbosuppressed processes.

The analysis of charmed particle decays has shown that flavor $\mathrm{SU}(3)$ symmetry is qualitatively obeyed, but important symmetry-breaking effects can be identified [3]. As one example, an argument using the U-spin subgroup of $\mathrm{SU}(3)$ predicts the rates for $D^{0} \rightarrow \pi^{+} \pi^{-}$ and $D^{0} \rightarrow K^{+} K^{-}$to be equal, but they differ by a factor of about three. This effect can be understood on the basis of SU(3)-breaking in decay constants and form factors [4].

Some interesting opportunities and questions have arisen recently in the context of singlyCabibbo-suppressed charm decays. Through the excellent photon and charged particle identification capabilities of the CLEO detector, it has become feasible to study many decays involving $\eta$ and $\eta^{\prime}$ [5]. The FOCUS Collaboration has recently amassed a large sample of charmed particles produced by high-energy photons at the Fermilab Tevatron [6]. Close and Lipkin [7] have recently identified some puzzles in this sector, including claims for rates for $D^{+} \rightarrow K^{*+} \bar{K}^{0}$ \& and $D^{+} \rightarrow K^{*+} \bar{K}^{* 0}$ [9] of the same order as some Cabibbo-favored two-body modes. We shall show that it is difficult to understand the first of these. While we do not have enough information to analyze charmed particle decays to VV, we use U-spin to relate these last two rates to those for $D_{s}^{+} \rightarrow \rho^{+} K^{0}$ and $D_{s}^{+} \rightarrow \rho^{+} K^{* 0}$, respectively, which should also have large rates if the claims are correct and approximate flavor symmetry is valid.

We recall notation in Section [1], and update results of Ref. [1] for Cabibbo-favored decays in Sec. [II]. We then tabulate results for Cabibbo-suppressed decays, and discuss specific relations among these decays (and between them and some Cabibbo-favored processes) in

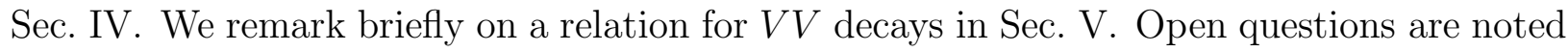
in Sec. VI, which concludes. 


\section{NOTATION}

We use the following quark content and phase conventions:

- Charmed mesons: $D^{0}=-c \bar{u}, D^{+}=c \bar{d}, D_{s}^{+}=c \bar{s}$;

- Pseudoscalar mesons: $\pi^{+}=u \bar{d}, \pi^{0}=(d \bar{d}-u \bar{u}) / \sqrt{2}, \pi^{-}=-d \bar{u}, K^{+}=u \bar{s}, K^{0}=d \bar{s}$, $\bar{K}^{0}=s \bar{d}, K^{-}=-s \bar{u}, \eta=(s \bar{s}-u \bar{u}-d \bar{d}) / \sqrt{3}, \eta^{\prime}=(u \bar{u}+d \bar{d}+2 s \bar{s}) / \sqrt{6} ;$

- Vector mesons: $\rho^{+}=u \bar{d}, \rho^{0}=(d \bar{d}-u \bar{u}) / \sqrt{2}, \rho^{-}=-d \bar{u}, \omega=(u \bar{u}+d \bar{d}) / \sqrt{2}, K^{*+}=u \bar{s}$, $K^{* 0}=d \bar{s}, \bar{K}^{* 0}=s \bar{d}, K^{*-}=-s \bar{u}, \phi=s \bar{s}$.

We denote the tree, color-suppressed, exchange, and annihilation amplitudes by $T, C$, $E$, and $A$, respectively. The exchange and annihilation diagrams that involve singlet contributions are labeled by $S E$ and $S A$. Penguin diagrams should be negligible since $V_{c d}^{*} V_{u d} \simeq-V_{c s}^{*} V_{u s}$. For the $P V$ modes, we use the subscripts $P$ and $V$ to refer to those diagrams with the spectator quark going into a pseudoscalar meson and a vector meson in the final state, respectively. To distinguish between Cabibbo-favored and Cabibbo-suppressed decay modes, the amplitudes associated with the former are all unprimed, while those with the latter are primed.

The partial width $\Gamma$ for a specific two-body decay to $P P$ is expressed in terms of an invariant amplitude $\mathcal{A}$ as

$$
\Gamma(H \rightarrow P P)=\frac{p^{*}}{8 \pi M_{H}^{2}}|\mathcal{A}|^{2},
$$

where $p^{*}$ is the center-of-mass (c.m.) 3-momentum of each final particle, and $M_{H}$ is the

mass of the decaying particle. The kinematic factor of $p^{*}$ is appropriate for the S-wave final state. The amplitude $\mathcal{A}$ will thus have a dimension of (energy). For $P V$ decays, on the other hand, a P-wave kinematic factor is appropriate instead, and

$$
\Gamma(H \rightarrow P V)=\frac{p^{* 3}}{8 \pi M_{H}^{2}}|\mathcal{A}|^{2} .
$$

In this case, $\mathcal{A}$ is dimensionless.

In the numerical calculation, we use for the charmed mesons $M_{D^{+}}=1.8693 \pm 0.0005 \mathrm{GeV}$ with $\tau\left(D^{+}\right)=1051 \pm 13$ fs, $M_{D^{0}}=1.8645 \pm 0.0005 \mathrm{GeV}$ with $\tau\left(D^{0}\right)=411.7 \pm 2.7$ fs, and $M_{D_{s}^{+}}=1.9685 \pm 0.0006 \mathrm{GeV}$ with $\tau\left(D_{s}^{+}\right)=490 \pm 9$ fs 10 . 
TABLE I: Branching ratios and invariant amplitudes for Cabibbo-favored decays of charmed mesons to two pseudoscalar mesons.

\begin{tabular}{|c|c|c|c|c|c|}
\hline Meson & Decay Mode & Representation & $\mathcal{B} 110$ & $p^{*}$ & $|\mathcal{A}|$ \\
\hline & & & $(\%)$ & $(\mathrm{MeV})$ & $\left(10^{-6} \mathrm{GeV}\right)$ \\
\hline \multirow[t]{4}{*}{$D^{0}$} & $K^{-} \pi^{+}$ & $T+E$ & $3.80 \pm 0.09$ & 861 & $2.48 \pm 0.03$ \\
\hline & $\bar{K}^{0} \pi^{0}$ & $\frac{1}{\sqrt{2}}(C-E)$ & $2.28 \pm 0.22$ & 860 & $1.92 \pm 0.09$ \\
\hline & $\bar{K}^{0} \eta$ & $C / \sqrt{3}$ & $0.76 \pm 0.11$ & 772 & $1.17 \pm 0.08$ \\
\hline & $\bar{K}^{0} \eta^{\prime}$ & $-\frac{1}{\sqrt{6}}(C+3 E)$ & $1.87 \pm 0.28$ & 565 & $2.15 \pm 0.16$ \\
\hline$D^{+}$ & $\bar{K}^{0} \pi^{+}$ & $C+T$ & $2.77 \pm 0.18$ & 862 & $1.33 \pm 0.04$ \\
\hline \multirow[t]{3}{*}{$D_{s}^{+}$} & $\bar{K}^{0} K^{+}$ & $C+A$ & $3.6 \pm 1.1$ & 850 & $2.35 \pm 0.36$ \\
\hline & $\pi^{+} \eta$ & $\frac{1}{\sqrt{3}}(T-2 A)$ & $1.7 \pm 0.5$ & 902 & $1.57 \pm 0.23$ \\
\hline & $\pi^{+} \eta^{\prime}$ & $\frac{2}{\sqrt{6}}(T+A)$ & $3.9 \pm 1.0$ & 743 & $2.62 \pm 0.34$ \\
\hline
\end{tabular}

\section{CABIBBO-FAVORED DECAYS}

In Tables If and II] we summarize predicted and observed amplitudes for Cabibbo-favored decays of charmed mesons to $P P$ and $P V$. The experimental values are based on Ref. [10] and supersede those quoted in Ref. [1]]. We then extract amplitudes for specific flavor topologies and their relative phases. Only the preferred solutions in Ref. [1] with updated data analysis are quoted in Table III. These parameters are needed since we will be using flavor SU(3) to relate them to the singly-Cabibbo-suppressed decays. For the sign of relative strong phases, we use the convention that $\delta_{A B}$ means the angle subtended from the amplitude $B$ to $A$. Using the relation $E_{V}=-E_{P}$ for the $P V$ modes in Table III, one would get the following strong phases for the last three amplitudes, all relative to $T_{V}$,

$$
\delta_{T_{P} T_{V}}=(-3 \pm 25)^{\circ}, \quad \delta_{C_{V} T_{V}}=(-170 \pm 13)^{\circ}, \quad \delta_{E_{V} T_{V}}=(-92 \pm 11)^{\circ} .
$$

It is interesting to observe that in this case $T_{P}$ and $T_{V}$ are roughly pointing in the same direction on the complex plane. The same is also true for $C_{P}$ and $C_{V}$, but pointing in almost the opposite direction to that of $T_{P}$ and $T_{V} . E_{P}$ and $E_{V}$, on the other hand, are close to $90^{\circ}$ from the above line. 
TABLE II: Branching ratios and invariant amplitudes for Cabibbo-favored decays of charmed mesons to one pseudoscalar and one vector meson.

\begin{tabular}{|c|c|c|c|c|c|}
\hline Meson & Decay Mode & Representation & 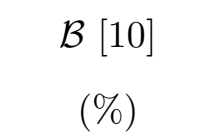 & $\begin{array}{c}p^{*} \\
(\mathrm{MeV})\end{array}$ & $\begin{array}{c}|\mathcal{A}| \\
\left(10^{-6}\right)\end{array}$ \\
\hline \multirow[t]{8}{*}{$D^{0}$} & $K^{*-} \pi^{+}$ & $T_{V}+E_{P}$ & $6.0 \pm 0.5$ & 711 & $4.83 \pm 0.20$ \\
\hline & $K^{-} \rho^{+}$ & $T_{P}+E_{V}$ & $10.2 \pm 0.8$ & 678 & $6.76 \pm 0.26$ \\
\hline & $\bar{K}^{* 0} \pi^{0}$ & $\frac{1}{\sqrt{2}}\left(C_{P}-E_{P}\right)$ & $2.8 \pm 0.4$ & 709 & $3.31 \pm 0.24$ \\
\hline & $\bar{K}^{0} \rho^{0}$ & $\left(C_{V}-E_{V}\right) / \sqrt{2}$ & $1.47 \pm 0.29$ & 676 & $2.57 \pm 0.25$ \\
\hline & $\bar{K}^{* 0} \eta$ & $\frac{1}{\sqrt{3}}\left(C_{P}+E_{P}-E_{V}\right)$ & $1.8 \pm 0.4$ & 580 & $3.59 \pm 0.40$ \\
\hline & $\bar{K}^{* 0} \eta^{\prime}$ & $-\frac{1}{\sqrt{6}}\left(C_{P}+E_{P}+2 E_{V}\right)$ & $<0.10$ & 99 & $<11.9$ \\
\hline & $\bar{K}^{0} \omega$ & $-\frac{1}{\sqrt{2}}\left(C_{V}+E_{V}\right)$ & $2.2 \pm 0.4$ & 670 & $3.20 \pm 0.29$ \\
\hline & $\bar{K}^{0} \phi$ & $-E_{P}$ & $0.94 \pm 0.11$ & 520 & $3.05 \pm 0.18$ \\
\hline \multirow[t]{2}{*}{$D^{+}$} & $\bar{K}^{* 0} \pi^{+}$ & $T_{V}+C_{P}$ & $1.92 \pm 0.19$ & 712 & $1.71 \pm 0.08$ \\
\hline & $\bar{K}^{0} \rho^{+}$ & $T_{P}+C_{V}$ & $6.6 \pm 2.5$ & 680 & $3.40 \pm 0.64$ \\
\hline \multirow[t]{7}{*}{$D_{s}^{+}$} & $\bar{K}^{* 0} K^{+}$ & $C_{P}+A_{V}$ & $3.3 \pm 0.9$ & 682 & $3.69 \pm 0.50$ \\
\hline & $\bar{K}^{0} K^{*+}$ & $C_{V}+A_{P}$ & $4.3 \pm 1.4$ & 683 & $4.20 \pm 0.68$ \\
\hline & $\rho^{+} \eta$ & $\frac{1}{\sqrt{3}}\left(T_{P}-A_{P}-A_{V}\right)$ & $10.8 \pm 3.1$ & 727 & $6.06 \pm 0.87$ \\
\hline & $\rho^{+} \eta^{\prime}$ & $\frac{1}{\sqrt{6}}\left(2 T_{P}+A_{P}+A_{V}\right)$ & $10.1 \pm 2.8$ & 470 & $11.3 \pm 1.6$ \\
\hline & $\pi^{+} \rho^{0}$ & $\frac{1}{\sqrt{2}}\left(A_{V}-A_{P}\right)$ & $<0.07$ & 827 & $<0.40$ \\
\hline & $\pi^{+} \omega$ & $\frac{1}{\sqrt{2}}\left(A_{V}+A_{P}\right)$ & $0.28 \pm 0.11$ & 822 & $0.81 \pm 0.16$ \\
\hline & $\pi^{+} \phi$ & $T_{V}$ & $3.6 \pm 0.9$ & 712 & $3.61 \pm 0.45$ \\
\hline
\end{tabular}

In Ref. [1] we did not fit amplitudes involving the annihilation terms $A_{P}$ and $A_{V}$. Normally we would have expected that $A_{P}=-A_{V}$ and hence that the decay $D^{+} \rightarrow \pi^{+} \omega$ would be suppressed while $D^{+} \rightarrow \pi^{+} \rho^{0}$ would provide information on the magnitude of $A_{P}$. This pattern was anticipated some time ago by Lipkin on the basis of a G-parity argument [11]. Instead, it is the latter decay which appears to be suppressed, while the former occurs with a measurable rate. It may be that the $\omega$ contains a small admixture of strange quarks, which would permit it to be produced via a $T_{V}$ amplitude, or rescattering effects could induce annihilation-like terms not respecting $A_{P}=-A_{V}$. Other Cabibbo-favored processes not 
TABLE III: Preferred solutions of magnitudes and relative phases of the invariant amplitudes for the Cabibbo-favored decay modes.

\begin{tabular}{ccc}
\hline \hline Amplitude & Magnitude & Relative Strong Phase \\
$P P$ & $\left(10^{-6} \mathrm{GeV}\right)$ & - \\
\hline$T$ & $2.67 \pm 0.20$ & $\delta_{C T}=(-151 \pm 4)^{\circ}$ \\
$C$ & $2.03 \pm 0.15$ & - \\
$E$ & $1.67 \pm 0.13$ & $\delta_{E T}=(115 \pm 5)^{\circ}$ \\
$A$ & $1.05 \pm 0.52$ & $\delta_{A T}=(-65 \pm 30)^{\circ}$ \\
\hline \hline$P V$ & $\left(10^{-6}\right)$ & \\
\hline$T_{V}$ & $3.61 \pm 0.45$ & \\
$C_{P}$ & $2.44 \pm 0.52$ & $\delta_{C_{P} T_{V}}=(-156 \pm 12)^{\circ}$ \\
$E_{P}$ & $3.05 \pm 0.18$ & $\delta_{E_{P} T_{V}}=(88 \pm 11)^{\circ}$ \\
\hline$T_{P}$ & $6.03 \pm 1.15$ & \\
$C_{V}$ & $2.74 \pm 0.46$ & $\delta_{C_{V} T_{P}}=(-168 \pm 24)^{\circ}$ \\
$E_{V}$ & $3.05 \pm 0.18$ & $\delta_{E_{V} T_{P}}=(-90 \pm 22)^{\circ}$ \\
\hline \hline
\end{tabular}

${ }^{a}$ Another possible solution is $\left|T_{P}\right|=(4.46 \pm 1.19) \times 10^{-6}$. It is disfavored because it gives an even unacceptably larger lower bound on $\left|A_{P}^{\prime}\right|$, as will be explained toward the end of Sec. IV B.

fitted in this scheme [1] include the decays $D_{s}^{+} \rightarrow \rho^{+} \eta$ and $D_{s}^{+} \rightarrow \rho^{+} \eta^{\prime}$.

\section{SINGLY-CABIBBO-SUPPRESSED DECAYS}

The topological amplitude decomposition of singly-Cabibbo-suppressed two-body $D$ decays is listed in Table $\mathbb{I V}(P P$ modes $)$ and $\mathrm{V}(P V$ modes $)$, where the relations $E_{V}^{\prime}=-E_{P}^{\prime}$ and $A_{V}^{\prime}=-A_{P}^{\prime}$ have been used.

\section{A. U-spin relations}

A number of relations between singly-Cabibbo-suppressed amplitudes follow from the U-spin symmetry interchanging $s$ and $d$ quarks [4, 12]. The effective interactions inducing the transitions $c \rightarrow s u \bar{s}$ and $c \rightarrow d u \bar{d}$ occur with equal and opposite CKM factors, leading 
TABLE IV: Branching ratios and invariant amplitudes for singly-Cabibbo-suppressed decays of charmed mesons to two pseudoscalar mesons.

\begin{tabular}{|c|c|c|c|c|c|}
\hline Meson & Decay Mode & Representation & $\begin{array}{c}\mathcal{B}[10] \\
\left(\times 10^{-3}\right)\end{array}$ & $\begin{array}{c}p^{*} \\
(\mathrm{MeV})\end{array}$ & $\begin{array}{c}|\mathcal{A}| \\
\left(10^{-7} \mathrm{GeV}\right)\end{array}$ \\
\hline \multirow[t]{8}{*}{$D^{0}$} & $\pi^{+} \pi^{-}$ & $-\left(T^{\prime}+E^{\prime}\right)$ & $1.43 \pm 0.07$ & 922 & $4.66 \pm 0.11$ \\
\hline & $\pi^{0} \pi^{0}$ & $-\frac{1}{\sqrt{2}}\left(C^{\prime}-E^{\prime}\right)$ & $0.84 \pm 0.22$ & 922 & $3.57 \pm 0.47$ \\
\hline & $K^{+} K^{-}$ & $T^{\prime}+E^{\prime}$ & $4.12 \pm 0.14$ & 791 & $8.53 \pm 0.14$ \\
\hline & $K^{0} \bar{K}^{0}$ & 0 & $0.71 \pm 0.19$ & 788 & $3.55 \pm 0.47$ \\
\hline & $\pi^{0} \eta$ & $\frac{1}{\sqrt{6}}\left(C^{\prime}-2 E^{\prime}-S E^{\prime}\right)$ & - & 846 & - \\
\hline & $\pi^{0} \eta^{\prime}$ & $\frac{1}{\sqrt{3}}\left(C^{\prime}+E^{\prime}+2 S E^{\prime}\right)$ & - & 678 & - \\
\hline & $\eta \eta$ & $\frac{2 \sqrt{2}}{3}\left(C^{\prime}+S E^{\prime}\right)$ & - & 755 & - \\
\hline & $\eta \eta^{\prime}$ & $-\frac{1}{3 \sqrt{2}}\left(C^{\prime}+6 E^{\prime}+7 S E^{\prime}\right)$ & - & 537 & - \\
\hline \multirow[t]{4}{*}{$D^{+}$} & $\pi^{+} \pi^{0}$ & $-\frac{1}{\sqrt{2}}\left(T^{\prime}+C^{\prime}\right)$ & $2.5 \pm 0.7$ & 925 & $3.86 \pm 0.54$ \\
\hline & $\pi^{+} \eta$ & $\frac{1}{\sqrt{3}}\left(T^{\prime}+2 C^{\prime}+2 A^{\prime}+S A^{\prime}\right)$ & $3.0 \pm 0.6$ & 848 & $4.41 \pm 0.44$ \\
\hline & $\pi^{+} \eta^{\prime}$ & $-\frac{1}{\sqrt{6}}\left(T^{\prime}-C^{\prime}+2 A^{\prime}+4 S A^{\prime}\right)$ & $5.0 \pm 1.0$ & 680 & $6.36 \pm 0.64$ \\
\hline & $K^{+} \bar{K}^{0}$ & $T^{\prime}-A^{\prime}$ & $5.8 \pm 0.6$ & 792 & $6.34 \pm 0.33$ \\
\hline \multirow[t]{4}{*}{$D_{s}^{+}$} & $\pi^{+} K^{0}$ & $-\left(T^{\prime}-A^{\prime}\right)$ & $<8$ & 916 & $<11$ \\
\hline & $\pi^{0} K^{+}$ & $-\frac{1}{\sqrt{2}}\left(C^{\prime}+A^{\prime}\right)$ & - & 917 & - \\
\hline & $\eta K^{+}$ & $\frac{1}{\sqrt{3}}\left(T^{\prime}+2 C^{\prime}-S A^{\prime}\right)$ & - & 835 & - \\
\hline & $\eta^{\prime} K^{+}$ & $\underline{\frac{1}{\sqrt{6}}\left(2 T^{\prime}+C^{\prime}+3 A^{\prime}+4 S A^{\prime}\right)}$ & 一 & 646 & - \\
\hline
\end{tabular}

to a term transforming as $U=1, U_{3}=0$. One then obtains the following relations:

PP decays:

$$
\begin{gathered}
\mathcal{A}\left(D^{0} \rightarrow \pi^{+} \pi^{-}\right)=-\mathcal{A}\left(D^{0} \rightarrow K^{+} K^{-}\right), \\
\mathcal{A}\left(D^{0} \rightarrow K^{0} \bar{K}^{0}\right)=0, \\
\mathcal{A}\left(D^{+} \rightarrow K^{+} \bar{K}^{0}\right)=-\mathcal{A}\left(D_{s}^{+} \rightarrow \pi^{+} K^{0}\right) .
\end{gathered}
$$

It has been known for some time that the relation (4) fails. The rate for $D^{0} \rightarrow K^{+} K^{-}$is about three times that for $D^{0} \rightarrow \pi^{+} \pi^{-}$. The corresponding amplitudes differ by a factor of 
TABLE V: Branching ratios and invariant amplitudes for singly-Cabibbo-suppressed decays of charmed mesons to one pseudoscalar and one vector meson.

\begin{tabular}{|c|c|c|c|c|c|}
\hline Meson & Decay Mode & Representation & $\begin{array}{c}\mathcal{B} 10 \\
\left(\times 10^{-3}\right)\end{array}$ & $\begin{array}{c}p^{*} \\
(\mathrm{MeV})\end{array}$ & $\begin{array}{c}|\mathcal{A}| \\
\left(10^{-7}\right) \\
\end{array}$ \\
\hline \multirow[t]{14}{*}{$\overline{D^{0}}$} & $\pi^{+} \rho^{-}$ & $-\left(T_{V}^{\prime}+E_{P}^{\prime}\right)$ & - & 766 & - \\
\hline & $\pi^{-} \rho^{+}$ & $-\left(T_{P}^{\prime}-E_{P}^{\prime}\right)$ & - & 766 & - \\
\hline & $\pi^{0} \rho^{0}$ & $-\frac{1}{2}\left(C_{P}^{\prime}+C_{V}^{\prime}\right)$ & - & 767 & - \\
\hline & $K^{+} K^{*-}$ & $T_{V}^{\prime}+E_{P}^{\prime}$ & $2.0 \pm 1.1$ & 610 & $11.11 \pm 3.05$ \\
\hline & $K^{-} K^{*+}$ & $T_{P}^{\prime}-E_{P}^{\prime}$ & $3.8 \pm 0.8$ & 610 & $15.31 \pm 1.61$ \\
\hline & $K^{0} \bar{K}^{* 0}$ & $-2 E_{P}^{\prime}$ & $<1.7$ & 605 & $<10.4$ \\
\hline & $\bar{K}^{0} K^{* 0}$ & $2 E_{P}^{\prime}$ & $<0.9$ & 605 & $<7.5$ \\
\hline & $\pi^{0} \omega$ & $\frac{1}{2}\left(C_{V}^{\prime}-C_{P}^{\prime}+2 S E_{P}^{\prime}\right)$ & - & 761 & - \\
\hline & $\pi^{0} \phi$ & $\frac{1}{\sqrt{2}}\left(C_{P}^{\prime}+S E_{P}^{\prime}\right)$ & $<1.4$ & 644 & $<8.5$ \\
\hline & $\eta \omega$ & $-\frac{1}{\sqrt{6}}\left(C_{P}^{\prime}+2 C_{V}^{\prime}+S E_{V}^{\prime}+4 S E_{P}^{\prime}\right)$ & - & 648 & - \\
\hline & $\eta^{\prime} \omega$ & $\frac{1}{2 \sqrt{3}}\left(C_{P}^{\prime}-C_{V}^{\prime}+4 S E_{V}^{\prime}-2 S E_{P}^{\prime}\right)$ & - & 333 & - \\
\hline & $\eta \phi$ & $\frac{1}{\sqrt{3}}\left(C_{P}^{\prime}-2 S E_{P}^{\prime}+S E_{V}^{\prime}\right)$ & $<2.8$ & 489 & $<18.3$ \\
\hline & $\eta \rho^{0}$ & $\frac{1}{\sqrt{6}}\left(2 C_{V}^{\prime}-C_{P}^{\prime}-S E_{V}^{\prime}\right)$ & - & 655 & - \\
\hline & $\eta^{\prime} \rho^{0}$ & $\frac{1}{2 \sqrt{3}}\left(C_{V}^{\prime}+C_{P}^{\prime}+4 S E_{V}^{\prime}\right)$ & - & 349 & - \\
\hline \multirow[t]{8}{*}{$D^{+}$} & $\pi^{+} \rho^{0}$ & $-\frac{1}{\sqrt{2}}\left(T_{V}^{\prime}+C_{P}^{\prime}-2 A_{P}^{\prime}\right)$ & $1.04 \pm 0.18$ & 769 & $3.55 \pm 0.31$ \\
\hline & $\pi^{0} \rho^{+}$ & $-\frac{1}{\sqrt{2}}\left(T_{P}^{\prime}+C_{V}^{\prime}+2 A_{P}^{\prime}\right)$ & - & 769 & - \\
\hline & $\pi^{+} \omega$ & $-\frac{1}{\sqrt{2}}\left(T_{V}^{\prime}+C_{P}^{\prime}+2 S A_{P}^{\prime}\right)$ & $<7$ & 763 & $<9.3$ \\
\hline & $\pi^{+} \phi$ & $C_{P}^{\prime}-S A_{P}^{\prime}$ & $6.1 \pm 0.6$ & 647 & $11.13 \pm 0.55$ \\
\hline & $\eta \rho^{+}$ & $\frac{1}{\sqrt{3}}\left(T_{P}^{\prime}+2 C_{V}^{\prime}+S A_{V}^{\prime}\right)$ & $<7$ & 659 & $<11.6$ \\
\hline & $\eta^{\prime} \rho^{+}$ & $-\frac{1}{\sqrt{6}}\left(T_{P}^{\prime}-C_{V}^{\prime}+4 S A_{V}^{\prime}\right)$ & $<5$ & 356 & $<24.7$ \\
\hline & $K^{+} \bar{K}^{* 0}$ & $T_{V}^{\prime}+A_{P}^{\prime}$ & $4.2 \pm 0.5$ & 610 & $10.08 \pm 0.60$ \\
\hline & $\bar{K}^{0} K^{*+}$ & $T_{P}^{\prime}-A_{P}^{\prime}$ & $31 \pm 14$ & 611 & $27.32 \pm 6.17$ \\
\hline \multirow[t]{8}{*}{$D_{s}^{+}$} & $\pi^{+} K^{* 0}$ & $-\left(T_{V}^{\prime}+A_{P}^{\prime}\right)$ & $6.5 \pm 2.8$ & 773 & $13.57 \pm 2.92$ \\
\hline & $\pi^{0} K^{*+}$ & $-\frac{1}{\sqrt{2}}\left(C_{V}^{\prime}-A_{P}^{\prime}\right)$ & - & 775 & - \\
\hline & $K^{+} \rho^{0}$ & $-\frac{1}{\sqrt{2}}\left(C_{P}^{\prime}+A_{P}^{\prime}\right)$ & $<2.9$ & 748 & $<9.5$ \\
\hline & $K^{0} \rho^{+}$ & $-\left(T_{P}^{\prime}-A_{P}^{\prime}\right)$ & - & 746 & - \\
\hline & $\eta K^{*+}$ & $\frac{1}{\sqrt{3}}\left(T_{P}^{\prime}+2 C_{V}^{\prime}+2 A_{P}^{\prime}-S A_{V}^{\prime}\right)$ & - & 661 & - \\
\hline & $\eta^{\prime} K^{*+}$ & $\frac{1}{\sqrt{6}}\left(2 T_{P}^{\prime}+C_{V}^{\prime}+A_{P}^{\prime}+4 S A_{V}^{\prime}\right)$ & - & 337 & - \\
\hline & $K^{+} \omega$ & $-\frac{1}{\sqrt{2}}\left(C_{P}^{\prime}-A_{P}^{\prime}-2 S A_{P}^{\prime}\right)$ & - & 741 & - \\
\hline & $K^{+} \phi$ & $T_{V}^{\prime}+C_{P}^{\prime}-A_{P}^{\prime}+S A_{P}^{\prime}$ & $<0.5$ & 607 & $<5.4$ \\
\hline
\end{tabular}


about 1.8, which can be ascribed to the product of a factor $f_{K} / f_{\pi} \simeq 1.22$ and a form factor ratio $F_{D \rightarrow K}\left(M_{K}^{2}\right) / F_{D \rightarrow \pi}\left(m_{\pi}^{2}\right) \simeq 1.5$ 田, 13]. Alternatively, it can be interpreted as saying that the subprocess $c \rightarrow s u \bar{s}$ leads to lower-multiplicity final states than $c \rightarrow d u \bar{d}$, since light quarks radiate extra pions easily. The $c \rightarrow s u \bar{s}$ subprocess responsible for $D^{0} \rightarrow K^{+} K^{-}$has only one light quark capable of radiating soft pions (in the current-algebra sense), whereas $c \rightarrow d u \bar{d}$ responsible for $D^{0} \rightarrow \pi^{+} \pi^{-}$has three such quarks. (The $\bar{u}$ spectator quark also can radiate soft pions in either case.) Therefore, one would expect the higher-multiplicity states to be more important in the fragmentation of the $c \rightarrow d u \bar{d}$ subprocess. We shall see presently that an estimate of the amplitude for $D^{0} \rightarrow \pi^{+} \pi^{-}$and $D^{0} \rightarrow K^{+} K^{-}$decays based on Cabibbo-favored decays lies between the experimental values for these decays.

The amplitude for $D^{0} \rightarrow K^{0} \bar{K}^{0}$ is predicted to vanish in the U-spin limit. Both the initial and final $(J=0)$ states have $U=0$, while the transition operator has $U=1$, as mentioned. The observed value of this amplitude is of the same order as the difference between the $D^{0} \rightarrow \pi^{+} \pi^{-}$and $D^{0} \rightarrow K^{+} K^{-}$amplitudes. Indeed, if one were to allow for different effective $c \rightarrow s u \bar{s}$ and $c \rightarrow d u \bar{d}$ transition strengths in the $E^{\prime}$ amplitudes alone, one would obtain the sum rule

$$
\mathcal{A}\left(D^{0} \rightarrow \pi^{+} \pi^{-}\right)+\mathcal{A}\left(D^{0} \rightarrow K^{+} K^{-}\right)+\mathcal{A}\left(D^{0} \rightarrow K^{0} \bar{K}^{0}\right)=0
$$

which is satisfied when the amplitudes are relatively real with respect to one another. However, there is no reason for (7) to hold in general. The decay $D^{0} \rightarrow K^{0} \bar{K}^{0}$ simply seems to occur at a level approriate for $\mathrm{SU}(3)$ symmetry breaking in other Cabibbo-suppressed $D^{0} \rightarrow P P$ decays.

The relation (6) is untested so far. It predicts a branching ratio $\mathcal{B}\left(D_{s}^{+} \rightarrow \pi^{+} K^{0}\right)=$ $(2.8 \pm 0.3) \times 10^{-3}$ on the basis of $\mathcal{B}\left(D^{+} \rightarrow K^{+} \bar{K}^{0}\right)=(5.8 \pm 0.6) \times 10^{-3}$ and kinematic correction factors. This should be an easy process to observe.

PV decays:

$$
\begin{gathered}
\mathcal{A}\left(D^{0} \rightarrow \pi^{+} \rho^{-}\right)=-\mathcal{A}\left(D^{0} \rightarrow K^{+} K^{*-}\right), \\
\mathcal{A}\left(D^{0} \rightarrow \pi^{-} \rho^{+}\right)=-\mathcal{A}\left(D^{0} \rightarrow K^{-} K^{*+}\right), \\
\mathcal{A}\left(D^{0} \rightarrow K^{0} \bar{K}^{* 0}\right)=-\mathcal{A}\left(D^{0} \rightarrow \bar{K}^{0} K^{* 0}\right), \\
\mathcal{A}\left(D^{+} \rightarrow K^{+} \bar{K}^{* 0}\right)=-\mathcal{A}\left(D_{s}^{+} \rightarrow \pi^{+} K^{* 0}\right),
\end{gathered}
$$




$$
\mathcal{A}\left(D^{+} \rightarrow \bar{K}^{0} K^{*+}\right)=-\mathcal{A}\left(D_{s}^{+} \rightarrow K^{0} \rho^{+}\right)
$$

The relations (8) and (9) are untested as yet because of the absence of $D^{0} \rightarrow \pi^{ \pm} \rho^{\mp}$ branching ratios. These processes should be observable in the CLEO-c detector. The relation (10) should be testable in the presence of an $E_{P}^{\prime}$ amplitude, whose magnitude we shall estimate presently. The relation (11) is satisfied within $1 \sigma$; it predicts $\mathcal{B}\left(D_{s}^{+} \rightarrow \pi^{+} K^{* 0}\right)=$ $(3.6 \pm 0.4) \times 10^{-3}$. Finally, the relation (12) is interesting since $\mathcal{B}\left(D^{+} \rightarrow \bar{K}^{0} K^{*+}\right)=(3.1 \pm$ $1.4) \%$ would entail a predicted branching ratio $\mathcal{B}\left(D_{s}^{+} \rightarrow K^{0} \rho^{+}\right)=(2.4 \pm 1.1) \%$. We shall see, however, that it is difficult to understand the large branching ratio for $D^{+} \rightarrow \bar{K}^{0} K^{*+}$ when extrapolating from the Cabibbo-favored $P V$ decays of charmed particles [7], even when one allows for the most favorable possible interference between contributing amplitudes.

\section{B. Relations between Cabibbo-favored and singly-Cabibbo-suppressed decays}

We now make use of the amplitudes determined in Ref. [1] and updated in Sec. III for Cabibbo-favored $P P$ and $P V$ decays to predict the magnitudes and phases of amplitudes for singly-Cabibbo-suppressed processes. We shall see that with the single exception of $D^{+} \rightarrow \bar{K}^{0} K^{*+}$, all results are consistent with a flavor $\mathrm{SU}(3)$ symmetry whose breaking does not exceed expected limits. The magnitudes of the topological amplitudes for singlyCabibbo-suppressed modes can be obtained from those for Cabibbo-favored ones listed in Table III by multiplying a Cabibbo suppression factor of $\lambda \simeq 0.2256$. We assume the relative strong phases stay the same. The resulting amplitudes are shown in Table $V 1$.

In the singly-Cabibbo-suppressed $P P$ decays, some modes can be directly related to their counterparts in the Cabibbo-favored ones. Assuming $S U(3)$ symmetry, we obtain

$$
\begin{aligned}
& \left|\mathcal{A}\left(D^{0} \rightarrow \pi^{+} \pi^{-}\right)\right|=\left|\mathcal{A}\left(D^{0} \rightarrow K^{+} K^{-}\right)\right|=\lambda\left|\mathcal{A}\left(D^{0} \rightarrow K^{-} \pi^{+}\right)\right| \simeq(5.60 \pm 0.07) \times 10^{-7} \mathrm{GeV} \\
& \left|\mathcal{A}\left(D^{0} \rightarrow \pi^{0} \pi^{0}\right)\right|=\lambda\left|\mathcal{A}\left(D^{0} \rightarrow \bar{K}^{0} \pi^{0}\right)\right| \simeq(4.34 \pm 0.21) \times 10^{-7} \mathrm{GeV}, \\
& \left|\mathcal{A}\left(D^{+} \rightarrow \pi^{+} \pi^{0}\right)\right|=\frac{\lambda}{\sqrt{2}}\left|\mathcal{A}\left(D^{+} \rightarrow \bar{K}^{0} \pi^{+}\right)\right| \simeq(2.12 \pm 0.07) \times 10^{-7} \mathrm{GeV}, \\
& \left|\mathcal{A}\left(D_{s}^{+} \rightarrow \pi^{0} K^{+}\right)\right|=\frac{\lambda}{\sqrt{2}}\left|\mathcal{A}\left(D_{s}^{+} \rightarrow \bar{K}^{0} K^{+}\right)\right| \simeq(3.76 \pm 0.57) \times 10^{-7} \mathrm{GeV} .
\end{aligned}
$$

Both $D^{+} \rightarrow K^{+} \bar{K}^{0}$ and $D_{s}^{+} \rightarrow \pi^{+} K^{0}$ involve the combination $T^{\prime}-A^{\prime}$, which does not have a counterpart in the Cabibbo-favored modes. Therefore, we use the values given in Table 
TABLE VI: Real and imaginary parts of the invariant amplitudes for the singly-Cabibbo-suppressed decay modes. It is assumed that $T^{\prime}$ and $T_{V}^{\prime}$ are purely real.

\begin{tabular}{cccccc}
\hline \hline $\begin{array}{c}\text { Amplitude } \\
P P\end{array}$ & $\begin{array}{c}\text { Re } \\
\left(10^{-7} \mathrm{GeV}\right)\end{array}$ & $\begin{array}{c}\text { Im } \\
\left(10^{-7} \mathrm{GeV}\right)\end{array}$ & $\begin{array}{c}\text { Amplitude } \\
\text { Re }\end{array}$ & $\begin{array}{c}\text { Im } \\
\left(10^{-7}\right)\end{array}$ & $\left(10^{-7}\right)$ \\
\hline$T^{\prime}$ & 6.02 & 0 & $T_{V}^{\prime}$ & 8.14 & 0 \\
$C^{\prime}$ & -4.01 & -2.22 & $C_{P}^{\prime}$ & -5.03 & -2.24 \\
$E^{\prime}$ & -1.59 & 3.41 & $E_{P}^{\prime}$ & 0.30 & 6.88 \\
$A^{\prime}$ & 1.00 & -2.15 & $T_{P}^{\prime}$ & 13.6 & -0.60 \\
& & & $C_{V}^{\prime}$ & -6.09 & -1.07 \\
& & & $E_{V}^{\prime}$ & -0.30 & -6.88 \\
\hline \hline
\end{tabular}

VI to estimate the magnitude of their amplitude:

$$
\left|\mathcal{A}\left(D^{+} \rightarrow K^{+} \bar{K}^{0}\right)\right|=\left|\mathcal{A}\left(D_{s}^{+} \rightarrow \pi^{+} K^{0}\right)\right| \simeq(5.47 \pm 1.30) \times 10^{-7} \mathrm{GeV}
$$

In the $P V$ decays, the following results are obtained:

$$
\begin{aligned}
& \left|\mathcal{A}\left(D^{0} \rightarrow \pi^{+} \rho^{-}\right)\right|=\left|\mathcal{A}\left(D^{0} \rightarrow K^{+} K^{*-}\right)\right|=\lambda\left|\mathcal{A}\left(D^{0} \rightarrow K^{*-} \pi^{+}\right)\right| \simeq(10.90 \pm 0.45) \times 10^{-7} \\
& \left|\mathcal{A}\left(D^{0} \rightarrow \pi^{-} \rho^{+}\right)\right|=\left|\mathcal{A}\left(D^{0} \rightarrow K^{-} K^{*+}\right)\right|=\lambda\left|\mathcal{A}\left(D^{0} \rightarrow K^{-} \rho^{+}\right)\right| \simeq(15.25 \pm 0.60) \times 10^{-7} \\
& \left|\mathcal{A}\left(D^{0} \rightarrow K^{0} \bar{K}^{* 0}\right)\right|=\left|\mathcal{A}\left(D^{0} \rightarrow \bar{K}^{0} K^{* 0}\right)\right|=2 \lambda\left|\mathcal{A}\left(D^{0} \rightarrow \bar{K}^{0} \phi\right)\right| \simeq(13.78 \pm 0.81) \times 10^{-7} \\
& \left|\mathcal{A}\left(D^{0} \rightarrow \pi^{0} \rho^{0}\right)\right| \simeq(5.83 \pm 0.78) \times 10^{-7}
\end{aligned}
$$

where the last line is computed directly using Table V1. It is seen that all the above predicted amplitude magnitudes agree well with those inferred from the measured branching ratios, apart from small differences that can be attributed to $S U(3)$ breaking. Table VII summarizes the comparison of predicted and experimental amplitudes.

An upper bound on $A_{P}$ can be extracted from Cabibbo-favored modes: $\left|A_{P}\right| \leq\left(\mid \mathcal{A}\left(D_{s}^{+} \rightarrow\right.\right.$ $\left.\left.\pi^{+} \rho^{0}\right)\left.\right|^{2}+\left|\mathcal{A}\left(D_{s}^{+} \rightarrow \pi^{+} \omega\right)\right|^{2}\right)^{1 / 2} \leq 1.4 \times 10^{-6}$ at the $3 \sigma$ level. This in turn implies that the corresponding singly-Cabibbo-suppressed amplitude satisfies

$$
\left|A_{P}^{\prime}\right|=\lambda\left|A_{P}\right| \leq 3.1 \times 10^{-7}
$$

Other information about the contribution of $A_{P}^{\prime}$ can be directly learned from, for example, the decay mode $D^{+} \rightarrow \pi^{+} \rho^{0}$. In order to reproduce the $1 \sigma$ lower limit on its amplitude, 
TABLE VII: Comparisons between predicted amplitudes based on Cabibbo-favored decays and the experimental values for singly-Cabibbo-suppressed decays of charmed mesons.

\begin{tabular}{cccc}
\hline \hline Meson Decay Mode & Prediction & Experimental value \\
& $P P$ & $\left(10^{-7} \mathrm{GeV}\right)$ & $\left(10^{-7} \mathrm{GeV}\right)$ \\
\hline$D^{0}$ & $\pi^{+} \pi^{-}$ & $5.60 \pm 0.07$ & $4.66 \pm 0.11$ \\
& $\pi^{0} \pi^{0}$ & $4.34 \pm 0.21$ & $3.57 \pm 0.47$ \\
& $K^{+} K^{-}$ & $5.60 \pm 0.07$ & $8.53 \pm 0.14$ \\
& $K^{0} \bar{K}^{0}$ & 0 & $3.55 \pm 0.47$ \\
\hline$D^{+}$ & $\pi^{+} \pi^{0}$ & $2.12 \pm 0.07$ & $3.86 \pm 0.54$ \\
& $K^{+} \bar{K}^{0}$ & $5.47 \pm 1.30$ & $6.34 \pm 0.33$ \\
\hline$D_{s}^{+}$ & $\pi^{+} K^{0}$ & $5.47 \pm 1.30$ & $<11$ \\
\hline \hline & $P V$ & $\left(10^{-7}\right)$ & $\left(10^{-7}\right)$ \\
\hline$D^{0}$ & $K^{+} K^{*-}$ & $10.90 \pm 0.45$ & $11.11 \pm 3.05$ \\
& $K^{-} K^{*+}$ & $15.25 \pm 0.60$ & $15.31 \pm 1.61$ \\
& $K^{0} \bar{K}^{* 0}$ & $13.78 \pm 0.81$ & $<10.4$ \\
& $\bar{K}^{0} K^{* 0}$ & $13.78 \pm 0.81$ & $<7.5$ \\
\hline \hline
\end{tabular}

using

$$
\left|\mathcal{A}\left(D^{+} \rightarrow \pi^{+} \rho^{0}\right)\right|=\left|\sqrt{2} A_{P}^{\prime}-\frac{\lambda}{\sqrt{2}} \mathcal{A}\left(D^{+} \rightarrow \bar{K}^{* 0} \pi^{+}\right)\right|,
$$

one must have $\left|A_{P}^{\prime}\right| \geq 0.36 \times 10^{-7}$, assuming that the two contributions interfere constructively. Since $\left|T_{V}^{\prime}\right| \simeq(8.2 \pm 2.6) \times 10^{-7}$, a small $\left|A_{P}^{\prime}\right|$ of about $2 \times 10^{-7}$ improves agreement with the extracted amplitude of $D^{+} \rightarrow K^{+} \bar{K}^{* 0}$, assuming constructive interference. However, there is trouble when one tries to interpret the experimental data for $D^{+} \rightarrow \bar{K}^{0} K^{*+}$. With $\left|T_{P}^{\prime}\right|=\lambda\left|T_{P}\right| \simeq(13.6 \pm 2.6) \times 10^{-7}$, one would need $\left|A_{P}^{\prime}\right| \gtrsim(13.7 \pm 6.7) \times 10^{-7}$ in order to reach the experimental result in Table $\nabla$, where the lower bound on $A_{P}^{\prime}$ assumes maximal constructive interference. This apparently contradicts the upper bound (13) obtained from the Cabibbo-favored modes. Since currently the branching ratio of $D^{+} \rightarrow \bar{K}^{0} K^{*+}$ is measured only at a level of slightly more than $2 \sigma$ [8], a definite conclusion cannot be drawn without more statistics. 


\section{Triangle and quadrangle relations}

From the PP modes listed in Table [V], one can find the following sum rules:

$$
\begin{aligned}
& \sqrt{2} \mathcal{A}\left(D^{+} \rightarrow \pi^{+} \pi^{0}\right)-\sqrt{2} \mathcal{A}\left(D^{0} \rightarrow \pi^{0} \pi^{0}\right)-\mathcal{A}\left(D^{0} \rightarrow \pi^{+} \pi^{-}\right)=0 \\
& \sqrt{2} \mathcal{A}\left(D^{+} \rightarrow \pi^{+} \pi^{0}\right)+\mathcal{A}\left(D^{+} \rightarrow K^{+} \bar{K}^{0}\right)-\sqrt{2} \mathcal{A}\left(D_{s}^{+} \rightarrow \pi^{0} K^{+}\right)=0, \\
& \sqrt{2} \mathcal{A}\left(D^{+} \rightarrow \pi^{+} \pi^{0}\right)-\mathcal{A}\left(D_{s}^{+} \rightarrow \pi^{+} K^{0}\right)-\sqrt{2} \mathcal{A}\left(D_{s}^{+} \rightarrow \pi^{0} K^{+}\right)=0, \\
& 2 \sqrt{2} \mathcal{A}\left(D^{+} \rightarrow \pi^{+} \eta\right)+\mathcal{A}\left(D^{+} \rightarrow \pi^{+} \eta^{\prime}\right)+\sqrt{6} \mathcal{A}\left(D^{+} \rightarrow K^{+} \bar{K}^{0}\right)+3 \sqrt{3} \mathcal{A}\left(D^{+} \rightarrow \pi^{+} \pi^{0}\right)=0 \\
& 2 \sqrt{2} \mathcal{A}\left(D_{s}^{+} \rightarrow \eta K^{+}\right)+\mathcal{A}\left(D_{s}^{+} \rightarrow \eta^{\prime} K^{+}\right)+\sqrt{6} \mathcal{A}\left(D_{s}^{+} \rightarrow \pi^{+} K^{0}\right)+3 \sqrt{3} \mathcal{A}\left(D_{s}^{+} \rightarrow \pi^{0} K^{+}\right)=0 .
\end{aligned}
$$

Moreover, any three amplitudes selected from $\mathcal{A}\left(D^{0} \rightarrow \pi^{0} \pi^{0}\right), \mathcal{A}\left(D^{0} \rightarrow \pi^{0} \eta\right), \mathcal{A}\left(D^{0} \rightarrow \pi^{0} \eta^{\prime}\right)$, $\mathcal{A}\left(D^{0} \rightarrow \eta \eta\right)$, and $\mathcal{A}\left(D^{0} \rightarrow \eta \eta^{\prime}\right)$ with appropriate coefficients can form a triangle.

\section{Decays involving $\eta$ and $\eta^{\prime}$}

The amplitudes for decays involving $\eta$ and $\eta^{\prime}$ contain unknown contributions corresponding to disconnected quark diagrams, such as $S E^{\prime}$ and $S A^{\prime}$ in the decays to $P P$. A satisfactory description of Cabibbo-favored decays to PP was obtained in Ref. [1] without the help of such contributions, but the Cabibbo-favored decays to $P V$ final states involving such contributions were not seen to follow a pattern describable through the flavor-topology approach. In the present subsection we discuss a test for the amplitudes $S E^{\prime}$ and $S A^{\prime}$ which can determine whether a flavor-topology description is suitable for singly-Cabibbosuppressed decays of charmed mesons to PP.

We express all amplitudes involving $\eta$ or $\eta^{\prime}$ in Table IV in terms of an unknown parameter $S E^{\prime}$ or $S A^{\prime}$ with unit coefficient: For example,

$$
\begin{gathered}
-\sqrt{6} \mathcal{A}\left(D^{0} \rightarrow \pi^{0} \eta^{\prime}\right)=2 E^{\prime}-C^{\prime}+S E^{\prime}, \\
\frac{\sqrt{3}}{2} \mathcal{A}\left(D^{0} \rightarrow \pi^{0} \eta^{\prime}\right)=\frac{1}{2}\left(C^{\prime}+E^{\prime}\right)+S E^{\prime}, \\
\frac{3}{2 \sqrt{2}} \mathcal{A}\left(D^{0} \rightarrow \eta \eta\right)=C^{\prime}+S E^{\prime}, \\
-\frac{3 \sqrt{2}}{7} \mathcal{A}\left(D^{0} \rightarrow \eta \eta^{\prime}\right)=\frac{1}{7}\left(C^{\prime}+6 E^{\prime}\right)+S E^{\prime},
\end{gathered}
$$

with four similar expressions (two for $D^{+}$and two for $D_{s}^{+}$) involving $S A^{\prime}$. Assuming $S E^{\prime}=$ $S A^{\prime}=0$ one can then plot these expressions in the complex plane, obtaining figures whose 
TABLE VIII: Complex amplitudes describing singly-Cabibbo-suppressed charmed meson decays to $P P$ involving $\eta$ and/or $\eta^{\prime}$. Real and imaginary parts of amplitudes are given in units of $10^{-6}$ $\mathrm{GeV}$. An additional unknown term $S E^{\prime}$ contributes to each of the first four decays and $S A^{\prime}$ to the last four.

\begin{tabular}{ccrr}
\hline \hline Amplitude & Expression & $\operatorname{Re}$ & $\operatorname{Im}$ \\
\hline$-\sqrt{6} \mathcal{A}\left(D^{0} \rightarrow \pi^{0} \eta\right)$ & $2 E^{\prime}-C^{\prime}$ & 0.082 & 0.905 \\
$-\frac{\sqrt{3}}{2} \mathcal{A}\left(D^{0} \rightarrow \pi^{0} \eta^{\prime}\right)$ & $\frac{1}{2}\left(C^{\prime}+E^{\prime}\right)$ & -0.280 & 0.060 \\
$\frac{3}{2 \sqrt{2}} \mathcal{A}\left(D^{0} \rightarrow \eta \eta\right)$ & $C^{\prime}$ & -0.401 & -0.222 \\
$-\frac{3 \sqrt{2}}{7} \mathcal{A}\left(D^{0} \rightarrow \eta \eta^{\prime}\right)$ & $\frac{1}{7}\left(C^{\prime}+6 E^{\prime}\right)$ & -0.194 & 0.261 \\
$\sqrt{3} \mathcal{A}\left(D^{+} \rightarrow \pi^{+} \eta\right)$ & $T^{\prime}+2 C^{\prime}+2 A^{\prime}$ & 0.001 & -0.873 \\
$-\frac{\sqrt{6}}{4} \mathcal{A}\left(D^{+} \rightarrow \pi^{+} \eta^{\prime}\right)$ & $\frac{1}{4}\left(T^{\prime}-C^{\prime}+2 A^{\prime}\right)$ & 0.301 & -0.052 \\
$-\sqrt{3} \mathcal{A}\left(D_{s}^{+} \rightarrow \eta K^{+}\right)$ & $-\left(T^{\prime}+2 C^{\prime}\right)$ & 0.199 & 0.444 \\
$\frac{\sqrt{6}}{4} \mathcal{A}\left(D_{s}^{+} \rightarrow \eta^{\prime} K^{+}\right)$ & $\frac{1}{4}\left(2 T^{\prime}+C^{\prime}+3 A^{\prime}\right)$ & 0.276 & -0.217 \\
\hline \hline
\end{tabular}

origins can be shifted by an amount corresponding to the unknown amplitude $S E^{\prime}$ or $S A^{\prime}$. The amplitudes plotted are summarized in Table VIII and described in Figs. 11 and 2.

The points for these $D^{0}$ decays all lie on a line, since the coefficients of $C^{\prime}$ and $E^{\prime}$ always sum to 1 . This is another way of expressing the linear dependence of the various decays mentioned in the previous subsection. In the case of $D^{+}$and $D_{s}^{+}$decays this linear dependence is not present.

The rates for $D^{+} \rightarrow \pi^{+} \eta$ and $D^{+} \rightarrow \pi^{+} \eta^{\prime}$ have been measured. Consequently, one may use them to draw circles about the corresponding points to search for common intersections. The line between each of these common intersection points and the origin corresponds to the complex amplitude $S A^{\prime}$ needed to reproduce the data. One solution corresponds to very small $S A^{\prime}$, while the other corresponds to a value comparable to the other amplitudes. Measurement of rates for $D_{s}^{+} \rightarrow \eta K^{+}$and $D_{s}^{+} \rightarrow \eta^{\prime} K^{+}$will permit a choice between these two solutions and a test of consistency of the description. A corresponding construction also will clearly be possible for $D^{0}$ decays once these are measured.

In principle similar techniques would be suitable for $P V$ decays. The decays $D^{0} \rightarrow \pi^{0} \omega$ and $D^{0} \rightarrow \pi^{0} \phi$ involve just the one unknown singlet amplitude $S E_{P}^{\prime}$, allowing a two-fold 


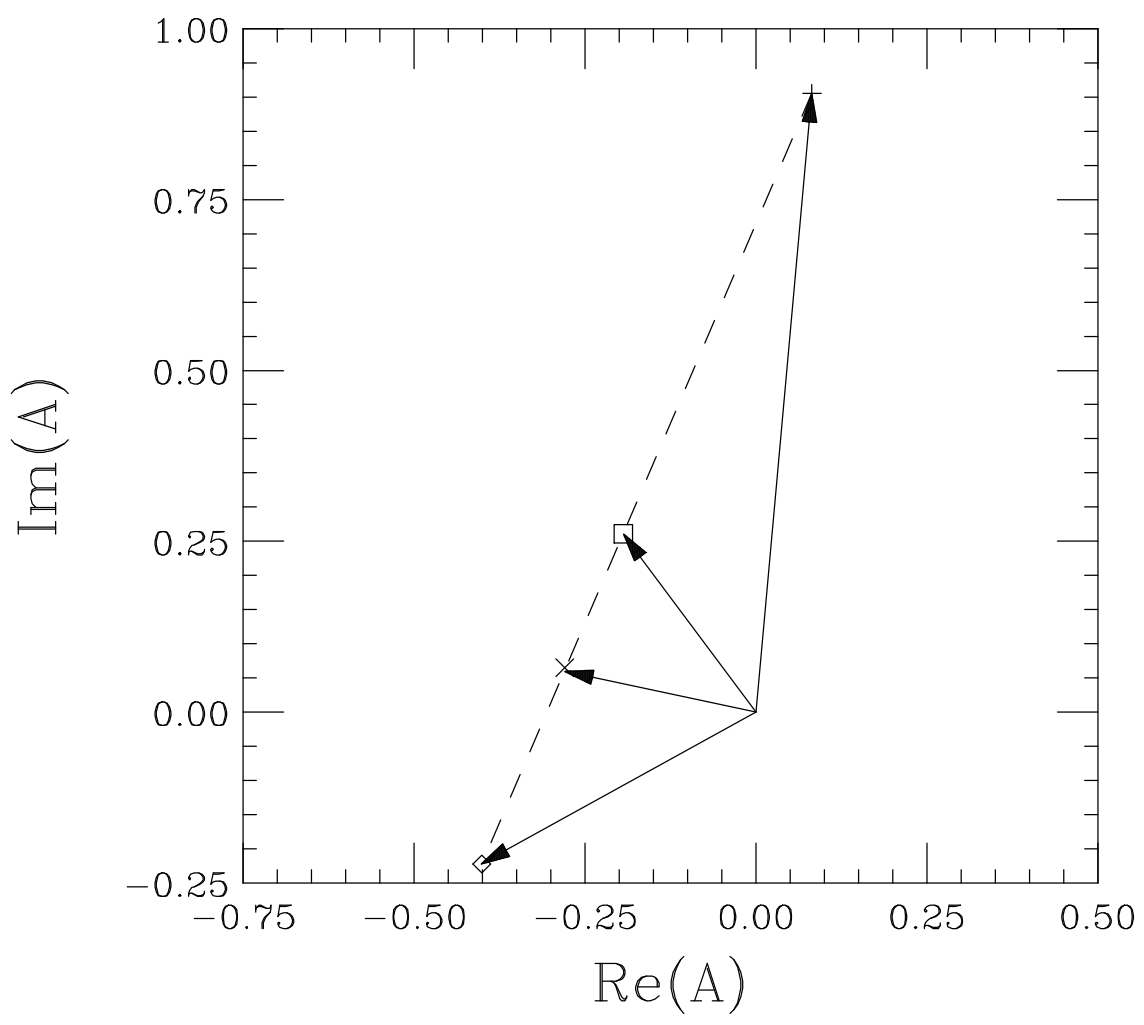

FIG. 1: Real and imaginary parts of amplitudes for $D^{0}$ decays to $P P$ final states involving $\eta$ and/or $\eta^{\prime}$. The origin may be shifted by an arbitrary amount $S E^{\prime} .+$ : $-\sqrt{6} \mathcal{A}\left(D^{0} \rightarrow \pi^{0} \eta\right) ; \times$ : $(\sqrt{3} / 2) \mathcal{A}\left(D^{0} \rightarrow \pi^{0} \eta^{\prime}\right) ; \diamond:(3 / 2 \sqrt{2}) \mathcal{A}\left(D^{0} \rightarrow \eta \eta\right) ; \square:-(3 \sqrt{2} / 7) \mathcal{A}\left(D^{0} \rightarrow \eta \eta^{\prime}\right)$.

solution in the manner of Fig. 2. Similarly, the decays $D^{0} \rightarrow \eta \rho^{0}$ and $D^{0} \rightarrow \eta^{\prime} \rho^{0}$ involve $S E_{V}^{\prime}$, and again there will be a two-fold solution. One can then test whether the four possible combinations of these solutions are compatible with the observed branching ratios for $D^{0} \rightarrow \eta \omega, D^{0} \rightarrow \eta^{\prime} \omega$, and $D^{0} \rightarrow \eta \phi$, which involve both $S E_{P}^{\prime}$ and $S E_{V}^{\prime}$.

In the case of $D^{+}$and $D_{s}^{+}$singly-Cabibbo-suppressed decays to $P V$, the presence of $A_{P}^{\prime}$ and $A_{V}^{\prime}$ in many amplitudes makes a similar program problematic. Without information on these quantities, which we found difficult to extract from Cabibbo-favored decays, the best one can do is to extract two possible solutions for $S A_{P}^{\prime}$ from the decay rates for $D^{+} \rightarrow \pi^{+} \omega$ and $D^{+} \rightarrow \pi^{+} \phi$, and two possible solutions for $S A_{V}^{\prime}$ from the decay rates for $D^{+} \rightarrow \eta \rho^{+}$ and $D^{+} \rightarrow \eta^{\prime} \rho^{+}$. One can, at least, see whether there is a need for disconnected diagrams in these processes. 


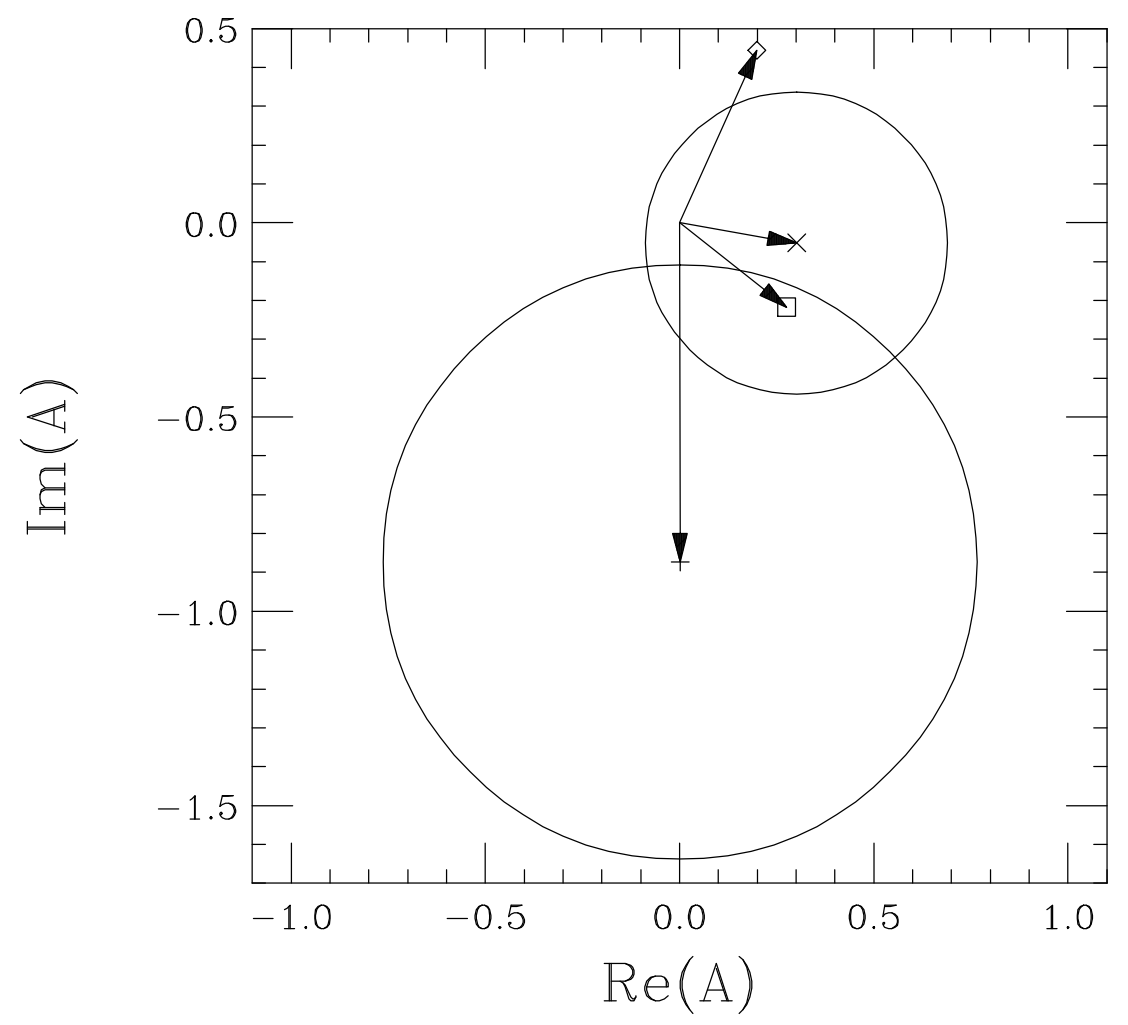

FIG. 2: Real and imaginary parts of amplitudes for $D^{+}$and $D_{s}^{+}$decays to $P P$ final states involving $\eta$ and/or $\eta^{\prime}$. The origin may be shifted by an arbitrary amount $S A^{\prime} .+: \sqrt{3} \mathcal{A}\left(D^{+} \rightarrow \pi^{+} \eta\right) ; \times$ : $-(\sqrt{6} / 4) \mathcal{A}\left(D^{+} \rightarrow \pi^{+} \eta^{\prime}\right) ; \diamond:-\sqrt{3} \mathcal{A}\left(D_{s}^{+} \rightarrow \eta K^{+}\right) ; \square:(\sqrt{6} / 4) \mathcal{A}\left(D_{s}^{+} \rightarrow \eta^{\prime} K^{+}\right)$. Circles about the points for $D^{+} \rightarrow \pi^{+} \eta$ and $D^{+} \rightarrow \pi^{+} \eta^{\prime}$ denote central values of the corresponding magnitudes.

\section{REMARKS ON $V V$ DECAYS}

The branching ratio for $D^{+} \rightarrow K^{*+} \bar{K}^{* 0}$ appears to be enhanced beyond that for a typical singly-Cabibbo-suppressed decay [7]. The amplitude relation

$$
\mathcal{A}\left(D^{+} \rightarrow K^{*+} \bar{K}^{* 0}\right)=-\mathcal{A}\left(D_{s}^{+} \rightarrow \rho^{+} K^{* 0}\right)
$$

should hold separately for each partial wave $(L=0,1,2)$ as a consequence of U-spin. The kinematic correction factors behave as $\left(p^{*}\right)^{2 L+1} / M_{H}^{2}$, so they cannot be directly applied unless we know the partial-wave decomposition of the decays. However, we can obtain a lower limit on the predicted branching ratio for $D_{s}^{+} \rightarrow \rho^{+} K^{* 0}$ by assuming that the decays are dominated by $L=0$. Given that $p^{*}=273 \mathrm{MeV}$ for the $D^{+}$decay and $524 \mathrm{MeV}$ for the $D_{s}^{+}$decay, we find that $\mathcal{B}\left(D^{+} \rightarrow K^{*+} \bar{K}^{* 0}\right)=(2.6 \pm 1.1) \%$ implies $\mathcal{B}\left(D_{s}^{+} \rightarrow \rho^{+} K^{* 0}\right)=$ $(2.1 \pm 0.9) \%$. If components with $L \neq 0$ are present this value becomes a lower bound. 


\section{OPEN QUESTIONS AND SUMMARY}

We have some evidence that the flavor-topology approach is limited in usefulness from the failure of the Cabibbo-favored decays $D_{s}^{+} \rightarrow \pi^{+} \rho^{0}$ and $D_{s}^{+} \rightarrow \pi^{+} \omega$ to fit any reasonble pattern for the amplitudes $A_{P}$ and $A_{V}$. Furthermore, it appears that disconnected quark diagrams, neglected in Ref. [1], appear necessary to fit the large branching ratios claimed for $D_{s}^{+} \rightarrow \pi^{+} \eta$ and $D_{s}^{+} \rightarrow \pi^{+} \eta^{\prime}$. One would expect analogues of these puzzles to appear in the singly-Cabibbo-suppressed decays. Certainly the processes $D^{+} \rightarrow K^{*+} \bar{K}^{0}$ and $D^{+} \rightarrow$

$K^{*+} \bar{K}^{* 0}$ noted by Close and Lipkin [7] are the most prominent candidates for such puzzles. It will be interesting to see the progress of future experimental studies, for example at CLEO-c, of these decays.

We have shown that aside from the two decays just noted, a reasonable description of $P P$ and $P V$ singly-Cabibbo-suppressed decays of charmed mesons appears possible by extrapolation from the Cabibbo-favored decays. As in the case of Cabibbo-favored decays, various amplitudes have non-trivial relative strong phases, indicating that these amplitudes are probably generated by final-state interactions governed by long-distance physics.

Decays involving $\eta$ and $\eta^{\prime}$ can be described if one is prepared to consider flavor topologies involving disconnected diagrams. The magnitudes of such amplitudes remain to be studied, but there are enough processes that once a few of them have beem measured, predictions will be possible for the remaining ones. Such studies bear the promise of useful insights on the strong interactions governing final-state interactions in charm decays, and may also shed indirect light on such interactions at the higher energies characterizing the decays of hadrons containing $b$ quarks.

\section{ACKNOWLEDGMENTS}

We thank H. J. Lipkin for discussions. J. L. R. is grateful to the Theory Group at Argonne National Laboratory for hospitality during part of this study. This work was supported in part by the United States Department of Energy, High Energy Physics Division, through Grant No. DE-FG02-90ER-40560 and Contract No. W-31-109-ENG-38. 
[1] J. L. Rosner, Phys. Rev. D 60, 114026 (1999) hep-ph/9905366.

[2] C. W. Chiang and J. L. Rosner, Phys. Rev. D 65, 054007 (2002) hep-ph/0110394.

[3] R. L. Kingsley, S. B. Treiman, F. Wilczek and A. Zee, Phys. Rev. D 11, 1919 (1975); M. B. Voloshin, V. I. Zakharov and L. B. Okun, Pis'ma v Zh. Eksp. Teor. Fiz. 21, 403 (1975) [Sov. Phys. - JETP Letters 21, 183 (1975) M. B. Einhorn and C. Quigg, Phys. Rev. D 12, 2015 (1975); C. Quigg, Z. Phys. C 4, 55 (1980); D. Zeppenfeld, Z. Phys. C 8, 77 (1981); L. L. Chau and H. Y. Cheng, Phys. Rev. D 36, 137 (1987); M. J. Savage and M. B. Wise, Phys. Rev. D 39, 3346 (1989) [Erratum-ibid. D 40, 3127 (1989)]; R. C. Verma and A. N. Kamal, Phys. Rev. D 43, 829 (1991); L. L. Chau, H. Y. Cheng, W. K. Sze, H. Yao and B. Tseng, Phys. Rev. D 43, 2176 (1991) [Erratum-ibid. D 58, 019902 (1998)], and references therein; A. Czarnecki, A. N. Kamal and Q. p. Xu, Z. Phys. C 54, 411 (1992); M. Gronau, O. F. Hernandez, D. London and J. L. Rosner, Phys. Rev. D 50, 4529 (1994) hep-ph/9404283; M. Gronau, O. F. Hernandez, D. London and J. L. Rosner, Phys. Rev. D 52, 6356 (1995) hep-ph/9504326.

[4] M. Gronau and J. L. Rosner, Phys. Lett. B 500, 247 (2001) hep-ph/0010237.

[5] E. von Toerne, private communication.

[6] See, for example, K. Stenson, talk at 9th International Symposium on Heavy Flavor Physics, Pasadena, California, 10-13 Sep 2001, hep-ex/0111083.

[7] F. E. Close and H. J. Lipkin, Argonne National Laboratory Report No. ANL-HEP-PR-02-070, hep-ph/0208217.

[8] P. L. Frabetti et al. [E687 Collaboration], Phys. Lett. B 346, 199 (1995).

[9] H. Albrecht et al. [ARGUS Collaboration], Z. Phys. C 53, 361 (1992).

[10] K. Hagiwara et al. [Particle Data Group Collaboration], Phys. Rev. D 66, 010001 (2002).

[11] H. J. Lipkin, Argonne National Laboratory Preprint No. ANL-HEP-CP-89-90, published in Heavy Quark Physics (Proceeding of the 1989 International Symposium on Heavy Quark Physics, Cornell University, Ithaca, NY), edited by P. S. Drell and D. L. Rubin, AIP Conference Proceedings No. 196 (New York, AIP, 1989), p. 72.

[12] M. Gronau, Phys. Lett. B 492, 297 (2000) hep-ph/0008292, and references therein.

[13] I. I. Bigi and A. I. Sanda, Phys. Lett. B 171, 320 (1986); L. L. Chau and H. Y. Cheng, Phys. Lett. B 333, 514 (1994) hep-ph/9404207. F. Buccella, M. Lusignoli, G. Miele, A. Pugliese and P. Santorelli, Phys. Rev. D 51, 3478 (1995) hep-ph/9411286. 